\title{
There is only one Fourier Transform
}

\section{Jens V. Fischer *}

\section{Microwaves and Radar Institute, German Aerospace Center (DLR), Germany}

\begin{abstract}
Four Fourier transforms are usually defined, the Integral Fourier transform, the Discrete-Time Fourier transform (DTFT), the Discrete Fourier transform (DFT) and the Integral Fourier transform for periodic functions. However, starting from their definitions, we show that all four Fourier transforms can be reduced to actually only one Fourier transform, the Fourier transform in the distributional sense.

\section{Keywords}

Integral Fourier Transform, Discrete-Time Fourier Transform (DTFT), Discrete Fourier Transform (DFT), Integral Fourier Transform for periodic functions, Fourier series, Poisson Summation Formula, periodization trick, interpolation trick
\end{abstract}

\section{Introduction}

The fact that "there is only one Fourier transform" actually needs no proof. It is commonly known and often discussed in the literature, for example in [1-2].

However frequently asked questions are: (1) What is the difference between calculating the Fourier series and the Fourier transform of a continuous function? (2) What is the difference between Discrete-Time Fourier Transform (DTFT) and Discrete Fourier Transform (DFT)? (3) When do we need which Fourier transform? and many others. The default answer today to all these questions is to invite the reader to have a look at the so-called Fourier-Poisson cube, e.g. in [2]. However, this advice may not be very helpful.

In this paper, in contrast to what has been done so far, we introduce two novel symbols, one $\amalg$ for discretization, i.e., to sample functions, and one $M$ for periodization, i.e., to create periodic functions from non-periodic ones. They will help us understanding the role of Poisson's Summation Formula in describing transitions from one Fourier transform variant to another. The overall intention of this study is to provide simple means to get all these things sorted and to help the reader to gain more confidence in dealing with finite and infinite Fourier series as well as with different Fourier transform variants. To keep things simple, we only treat the one-dimensional case $(t \in \mathbb{R})$. For $\mathrm{n}$-dimensional cases $\left(t \in \mathbb{R}^{n}, n>1\right)$ one may refer to $[3-5]$.

\section{Two Important Operations}

There are two operations which are being strongly related to four different variants of the Fourier transform, i.e., discretization and periodization. For any real-valued $\mathrm{T}>0$ and $\delta(t)$ being the Dirac impulse, let

$$
\left(\Perp_{T} f\right)(t):=\sum_{k=-\infty}^{\infty} f(k T) \delta(t-k T)
$$

be the function that results from a discretization of $f(t)$ and let

$$
\left({\aleph_{T}}_{T} f\right)(t):=\sum_{k=-\infty}^{\infty} f(t-k T)
$$

be the function that results from a periodization of $f(t)$, both defined as in [6-7]. Let us furthermore use four different Fourier transform definitions (see e.g. [2]), given in the Appendix. We then claim that starting from these definitions we are able to show that they reduce to

$$
\begin{aligned}
\mathcal{F}_{\text {per }}: & \mathcal{F}\left\{{\aleph_{T}}_{T} f(t)\right\} & =\frac{1}{T} \uplus_{\frac{1}{T}} \mathcal{F}\{f(t)\} \\
\text { DTFT : } & \frac{1}{T} \mathcal{F}\left\{\amalg_{\frac{1}{T}} f(t)\right\} & ={\aleph_{T}}_{T} \mathcal{F}\{f(t)\}
\end{aligned}
$$

and

$$
\begin{aligned}
& \text { DFT : } \quad \mathcal{F}\left\{\Perp \triangle_{N} f(t)\right\}=\frac{1}{N} \triangle \Perp_{\frac{1}{N}} \mathcal{F}\{f(t)\} \\
& \mathrm{DFF}^{-1}: \frac{1}{N} \mathcal{F}\left\{\triangle \Delta \Perp_{\frac{1}{N}} f(t)\right\}=\Perp \triangle_{N} \mathcal{F}\{f(t)\}
\end{aligned}
$$

with $\mathcal{F}$, the Fourier transform in the distributional sense [8].

*Corresponding author: Jens V. Fischer, Microwaves and Radar Institute, German Aerospace Center (DLR), Münchener Straße 20, 82234 Weßling, Germany, E-mail: jens.fischer@dlr.de 


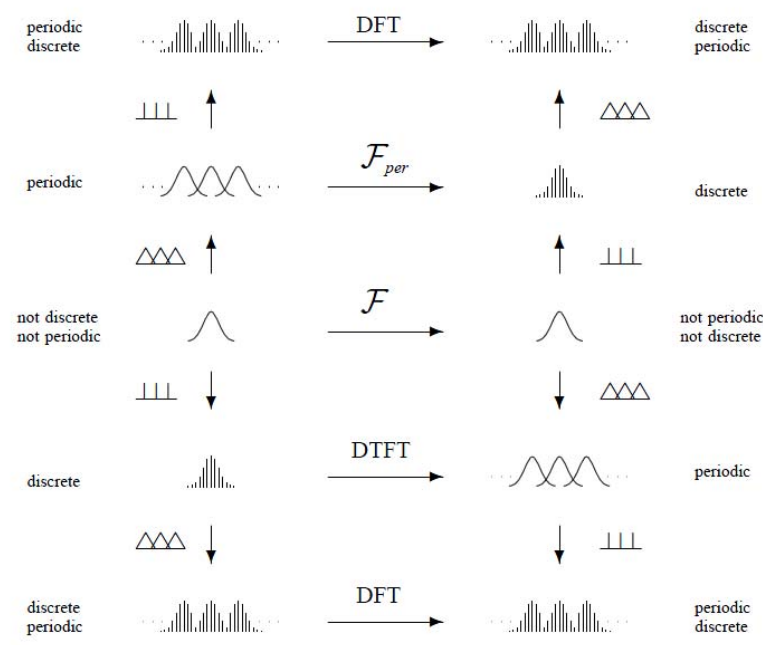

Figure 1: Four Fourier transforms: $\mathcal{F}, \mathcal{F}_{\text {per, }}$ DTFT and DFT, linked via operations of discretization $\amalg$ and periodization $M$. All four transforms coincide with $\mathcal{F}$ in the generalized functions sense. The diagram itself forms a Möbius strip.

These formulas are moreover rigorously based on generalized functions theory and part of a wider symbolic calculation scheme [3-4], mainly summarized in Table 1 and Table 2 and their relationship to four different Fourier transform variants, i.e., to $\mathcal{F}, \mathcal{F}_{\text {per, }}$ DTFT and DFT, is summarized in Table 3 and Table 4 . As a rule of thumb for their validity it is very useful to know that

- functions being discretized should not grow faster than polynomials and

- functions being periodized should decay to zero faster than polynomials,

otherwise they will cease to converge. These requirements are furthermore dual to each other in the sense that if a function fulfills one requirement then its Fourier transform fulfills the other [3-4].

\section{Derivations}

We will see in the following that defining the Integral Fourier Transform for periodic functions via (9) and (10) leads to (3), defining the Discrete-Time Fourier Transform (DTFT) via (11) and (12) leads to (4) and defining the Discrete Fourier Transform (DFT) via (13) and (14) leads to (5) and (6).

Most interesting is the fact that there has obviously been no other choice than defining all four Fourier transforms (see Appendix) exactly in this way. All their transitions reduce to Poisson's Summation Formula (Rule 1 and Rule 2 in Table 1) which obviously is the "glue" in-between these transforms. There is no scope whatsoever for possibly deviating definitions, i.e., all four transform definitions as summarized in the Appendix already follow from the definitions of $\mathcal{F}$ as well as (1) and (2).

The reason why equations (3)-(6) could not be found with conventional means (without generalized functions) is twofold. It results from the two (dual) facts that Lebesgue's integral is inadequate today, in the sense that

- it yields zero if discrete functions are integrated and

- $\quad$ it yields infinity if periodic functions are integrated.

These disadvantages have been overcome in generalized functions theory because it allows measuring discrete and periodic functions in the sense of Horváth's integral [9].



Figure 2: Four important domains: non-discrete non-periodic functions, periodic functions, discrete functions and discrete periodic functions; linked via discretization $\Perp$ and periodization $M$.

\section{Fourier Transform for Periodic Functions $\mathcal{F}_{\text {per }}$}

In this subsection we will see that if a periodic function is Fourier transformed via the Fourier transform for periodic functions (9) then the result is a discrete function. Indeed, inserting some $p(t)=\left(\Delta \triangle_{T} f\right)(t)$ into (9) yields

$$
\begin{aligned}
\hat{p}(m) & =\frac{1}{T} \int_{0}^{T} \Delta_{T} f(t) e^{-2 \pi i \frac{m}{T} t} d t \\
& =\frac{1}{T} \int_{-\infty}^{\infty} f(t) e^{-2 \pi i \frac{m}{T} t} d t \\
& =\frac{1}{T}(\mathcal{F} f)\left(\frac{m}{T}\right)
\end{aligned}
$$

where we used the popular periodization trick [10-17] and Fourier transform definition (7). Inserting these coefficients into (10) we obtain

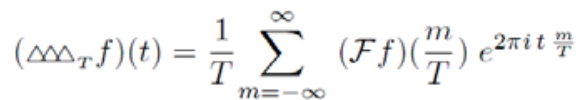

$$
\begin{aligned}
& =\frac{1}{T} \mathcal{F}^{-1}\left\{\sum_{m=-\infty}^{\infty}(\mathcal{F} f)\left(\frac{m}{T}\right) \delta\left(\sigma-\frac{m}{T}\right)\right\} \\
& =\frac{1}{T} \mathcal{F}^{-1}\left\{\left(\Psi_{\frac{1}{T}}(\mathcal{F} f)\right)(\sigma)\right\} \\
& =\frac{1}{T}\left(\mathcal{F}^{-1} \amalg_{\frac{1}{T}} \mathcal{F} f\right)(t)
\end{aligned}
$$

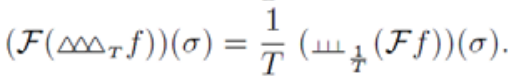

Therefore, (9) inserted into (10) reduces to

$$
\mathcal{F}\left({\aleph_{T}}_{T} f\right)=\frac{1}{T} \amalg_{\frac{1}{T}}(\mathcal{F} f)
$$

as a function of $\sigma \in \mathbb{R}$. This is formula (3). 
Table 1: Discrete Functions vs. Periodic Functions.

\begin{tabular}{|c|c|c|}
\hline No & Rule & Remark \\
\hline 1 & $\mathcal{F}\left(\amalg_{\frac{1}{T}} f\right)=T \Delta_{T}(\mathcal{F} f)$ & Poisson Sum Formula \\
\hline 2 & $\mathcal{F}\left(\Delta_{T} f\right)=\frac{1}{T} \amalg_{\frac{1}{T}}(\mathcal{F} f)$ & Poisson Sum Formula \\
\hline 3 & $\mathcal{F}(\amalg \amalg f)=\Delta \Delta(\mathcal{F} f)$ & abbreviated for $T=1$ \\
\hline 4 & $\mathcal{F}(\triangle \otimes f)=\Perp \amalg(\mathcal{F} f)$ & abbreviated for $T=1$ \\
\hline 5 & $\mathcal{F}(\Perp 1)=\Delta \triangle(\mathcal{F} 1)$ & where $\mathcal{F} 1=\delta$ \\
\hline 6 & $\mathcal{F}(\triangle \triangle \delta)=\Perp(\mathcal{F} \delta)$ & where $\mathcal{F} \delta=1$ \\
\hline 7 & $\mathcal{F}(\Perp 1)=\Perp 1$ & Dirac comb invariance \\
\hline 8 & $\mathcal{F}(\triangle \Delta \delta)=\triangle \triangle \delta$ & Dirac comb invariance \\
\hline 9 & $\Perp_{T} 1=\Delta_{T} \delta$ & Dirac comb identity \\
\hline 10 & $\triangle \Delta \delta=\Perp 1$ & Dirac comb identity \\
\hline 11 & $\mathcal{F}\left(\amalg_{\frac{1}{T}} 1\right)=T{\Delta_{T} \delta} \delta$ & Dirac comb reciprocity \\
\hline 12 & $\mathcal{F}\left(\triangle_{T} \delta\right)=\frac{1}{T} \amalg_{\frac{1}{T}} 1$ & Dirac comb reciprocity \\
\hline 13 & $\mathcal{F}(f \cdot g)=\mathcal{F} f * \mathcal{F} g$ & multiplication \\
\hline 14 & $\mathcal{F}(f * g)=\mathcal{F} f \cdot \mathcal{F} g$ & convolution \\
\hline 15 & $\amalg g \cdot f=\Perp(g \cdot f)$ & discretization \\
\hline 16 & $\Delta \triangle g * f=\Delta \Delta(g * f)$ & periodization \\
\hline 17 & $\amalg 1 \cdot f=\amalg(1 \cdot f)=\amalg f$ & discretization of $f$ \\
\hline 18 & $\Delta \Delta \delta * f=\Delta \Delta(\delta * f)=\Delta \Delta f$ & periodization of $f$ \\
\hline
\end{tabular}

where $\Perp \mathrm{f}$ is a discrete function sampled at integers and $\Delta \mathrm{f}$ is a one-periodic function

\section{Fourier Transform for Discrete Functions (DTFT)}

We show that if a discrete function is Fourier transformed via the DTFT then the result is a periodic function. As (11) is a Fourier series, it is periodic. The ansatz is therefore to

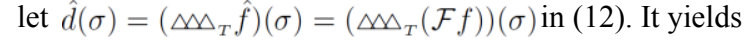

$$
\begin{aligned}
d(k) & =\int_{0}^{T}{\aleph_{T}}_{T} \hat{f}(\sigma) e^{2 \pi i \frac{k}{T} \sigma} d \sigma \\
& =\int_{-\infty}^{\infty} \hat{f}(\sigma) e^{2 \pi i \frac{k}{T} \sigma} d \sigma \\
& =\left(\mathcal{F}^{-1}(\mathcal{F} f)\right)\left(\frac{k}{T}\right)=f\left(\frac{k}{T}\right) .
\end{aligned}
$$

Inserting these coefficients into (11) we obtain

$$
\begin{aligned}
& \left({\aleph_{T}}_{T}(\mathcal{F} f)\right)(\sigma)=\frac{1}{T} \sum_{k=-\infty}^{\infty} f\left(\frac{k}{T}\right) e^{-2 \pi i \sigma \frac{k}{T}} \\
& =\frac{1}{T} \mathcal{F}\left\{\sum_{k=-\infty}^{\infty} f\left(\frac{k}{T}\right) \delta\left(t-\frac{k}{T}\right)\right\} \\
& =\frac{1}{T} \mathcal{F}\left\{\left(\Perp_{\frac{1}{T}} f\right)(t)\right\} \\
& =\frac{1}{T}\left(\mathcal{F}\left(\Psi_{\frac{1}{T}} f\right)\right)(\sigma) \text {. }
\end{aligned}
$$

Thus, (12) inserted into (11) reduces to

$$
\mathcal{F}\left(\amalg_{\frac{1}{T}} f\right)=T{\aleph_{T}}_{T}(\mathcal{F} f)
$$

as a function of $\sigma \in \mathbb{R}$. This is formula (4).

\section{Fourier Transform for Discrete Periodic Functions (DFT)}

\begin{tabular}{|c|c|c|}
\hline No & Rule & Remark \\
\hline $\mathrm{i}$ & $\mathcal{F}\left(\Perp_{\frac{1}{T}} \Delta \triangle_{P} f\right)=\frac{T}{P} \Delta \Delta_{T} \amalg_{\frac{1}{P}}(\mathcal{F} f)$ & Rule $1+2$ \\
\hline ii & $\mathcal{F}\left(\Delta \triangle_{P} \amalg_{\frac{1}{T}} f\right)=\frac{T}{P} \amalg_{\frac{1}{P}} \triangle_{T}(\mathcal{F} f)$ & Rule $2+1$ \\
\hline iii & $\amalg \Delta_{N} f=\aleph_{N} \amalg f$ & identity \\
\hline $\mathrm{v}$ & $\mathcal{F}\left(\amalg \Delta_{N} f\right)=\frac{1}{N} \aleph \amalg_{\frac{1}{N}}(\mathcal{F} f)$ & Rule $3+2$ \\
\hline vi & $\mathcal{F}\left(\Delta \amalg_{\frac{1}{N}} f\right)=N \Perp \Delta \Delta_{N}(\mathcal{F} f)$ & Rule $4+1$ \\
\hline
\end{tabular}

We show that if a discrete periodic function is Fourier transformed via the DFT, then the result is again a discrete periodic function. First, a simple reasoning tells us that after discretizing a periodic function $\aleph_{T} f(t)$ it can be denoted as $y(t)=\Perp \aleph_{N} f(t)$ where $N$ is an integer corresponding to its period $T$.
Table 2: Discrete Periodic Functions.

Inserting now its coefficients $\aleph_{N} f(k)$ with $k \in \mathbb{Z}$ into (13) yields



$$
\begin{aligned}
& =\frac{1}{N} \sum_{k=-\infty}^{\infty} f(k) e^{-2 \pi i \frac{m}{N} k} \\
& =\frac{1}{N}(\mathcal{F}(\Perp f))\left(\frac{m}{N}\right) \\
& =\frac{1}{N}(\bowtie \triangle(\mathcal{F} f))\left(\frac{m}{N}\right)
\end{aligned}
$$

using, again, the periodization trick (applied to a discrete function this time), definition (1) and a previous result. Inserting these coefficients into (14) we obtain

$$
\begin{aligned}
\left({\Delta_{N}}_{N} f\right)(k) & =\frac{1}{N} \sum_{m=0}^{N-1} \bowtie(\mathcal{F} f)\left(\frac{m}{N}\right) e^{2 \pi i k \frac{m}{N}} \\
& =\frac{1}{N} \sum_{m=-\infty}^{\infty}(\mathcal{F} f)\left(\frac{m}{N}\right) e^{2 \pi i k \frac{m}{N}} \\
& =\frac{1}{N}\left(\mathcal{F}^{-1} \Perp_{\frac{1}{N}}(\mathcal{F} f)\right)(k) .
\end{aligned}
$$

Thus, (13) inserted into (14) reduces to

$$
\begin{aligned}
\left(\triangle_{N} f\right)(k) & =\frac{1}{N} \quad\left(\mathcal{F}^{-1} \amalg_{\frac{1}{N}}(\mathcal{F} f)\right)(k), \quad k \in \mathbb{Z} \\
\left(\Perp \triangle_{N} f\right)(t) & =\frac{1}{N}\left(\Perp \mathcal{F}^{-1} \amalg_{\frac{1}{N}}(\mathcal{F} f)\right)(t), \quad t \in \mathbb{R}
\end{aligned}
$$

and with Rule 4 and Fourier transforming both sides it yields

$$
\mathcal{F}\left(\Perp \aleph_{N} f\right)=\frac{1}{N} \aleph_{\Perp_{\frac{1}{N}}}(\mathcal{F} f)
$$

as a function of $\sigma \in \mathbb{R}$.

\section{Derivation of the Inverse DFT}

As (13) is a Fourier series, it is periodic. Evaluating (14) for $\hat{g}(m)=\left({\triangle_{N}}_{N} \hat{f}\right)(m)$ where $\hat{f}=\mathcal{F} f$ yields

$$
\begin{aligned}
g(k) & =\sum_{m=0}^{N-1}\left({\triangle_{N}}_{N}(\mathcal{F} f)\right)(m) e^{2 \pi i \frac{k}{N} m} \\
& =\sum_{k=-\infty}^{\infty}(\mathcal{F} f)(m) e^{2 \pi i \frac{k}{N} m} \\
& =\left(\mathcal{F}^{-1} \Perp(\mathcal{F} f)\right)\left(\frac{k}{N}\right) \\
& =\left(\mathcal{F}^{-1} \mathcal{F}(\Delta \Delta f)\right)\left(\frac{k}{N}\right) \\
& =(\Delta \Delta f)\left(\frac{k}{N}\right)
\end{aligned}
$$

and inserting 
Table 3: Comparison of $\mathcal{F}, \mathcal{F}_{\text {per, DTFT and DFT }}$

\begin{tabular}{|c|c|c|c|}
\hline Transform & Input Function & Output Function & Rel. to $\mathcal{F}$ \\
\hline $\mathcal{F}$ & $f$ & $\hat{f}$ & identity \\
\hline $\mathcal{F}_{\text {per }}$ & $\Delta \Delta f$ & $\Perp \hat{f}$ & Rule 2 \\
\hline DTFT & $\Perp f f$ & $\Delta \Delta \hat{f}$ & Rule 1 \\
\hline DFT & $\Perp \Delta \Delta f$ & $\triangle \otimes \perp \perp \hat{f}$ & Rule iv \\
\hline $\mathcal{F}^{-1}$ & $\overline{\bar{f}}$ & $\overline{f f}$ & inverse \\
\hline $\mathcal{F}_{\text {per }}{ }^{-1}$ & $\Perp \hat{f}$ & $\Delta \Delta f$ & Rule 1 \\
\hline DTFT $^{-1}$ & $\Delta \Delta \hat{f}$ & $\Perp f$ & Rule 2 \\
\hline $\mathrm{DFT}^{-1}$ & $\triangle \triangle \perp \perp \hat{f}$ & $\perp \Perp \Delta \Delta f$ & Rule v \\
\hline
\end{tabular}

Table 4: Relations among $\mathcal{F}, \mathcal{F}_{\text {per, DTFT and DFT }}$

\begin{tabular}{|c|c|}
\hline Transform & Expressed in terms of $\mathcal{F}, \amalg, \bowtie$ \\
\hline $\mathcal{F}_{\text {per }} \equiv \mathrm{DTFT}^{-1}$ & $\mathcal{F} \circ \triangle \otimes=\Perp \circ \mathcal{F}$ \\
\hline DTFT $\equiv \mathcal{F}_{p e r}^{-1}$ & $\mathcal{F} \circ \Perp=\Delta \triangle \circ \mathcal{F}$ \\
\hline DFT & $\mathcal{F} \circ \amalg \circ \Delta \Delta=\triangle \Delta \circ \amalg \circ \mathcal{F}$ \\
\hline $\mathrm{DFT}^{-1}$ & $\mathcal{F} \circ \Delta \triangle \circ \amalg=\Perp \circ \Delta \Delta \circ \mathcal{F}$ \\
\hline
\end{tabular}

this result into (13) we obtain

$$
\begin{aligned}
\left(\triangle_{N}(\mathcal{F} f)\right)(m) & =\frac{1}{N} \sum_{k=0}^{N-1}(\bowtie f)\left(\frac{k}{N}\right) e^{-2 \pi i m \frac{k}{N}} \\
& =\frac{1}{N} \sum_{k=-\infty}^{\infty} f\left(\frac{k}{N}\right) e^{-2 \pi i m \frac{k}{N}} \\
& =\frac{1}{N}\left(\mathcal{F}\left(\Perp_{\frac{1}{N}} f\right)\right)(m) .
\end{aligned}
$$

Thus, (14) inserted into (13) reduces to

$$
\begin{aligned}
& \left(\mathcal{F}\left(\omega_{\frac{1}{N}} f\right)\right)(m)=N\left({\aleph_{N}}(\mathcal{F} f)\right)(m), \quad m \in \mathbb{Z} \\
& \left(\Perp \mathcal{F}\left(\Perp_{\frac{1}{v}} f\right)\right)(\sigma)=N\left(\Perp{\aleph_{N}}_{N}(\mathcal{F} f)\right)(\sigma), \quad \sigma \in \mathbb{R}
\end{aligned}
$$

and with Rule 4 on the left-hand side it yields

$$
\mathcal{F}\left(\triangle \Perp_{\frac{1}{N}} f\right)=N \Perp \triangle_{N}(\mathcal{F} f)
$$

as a function of $\sigma \in \mathbb{R}$.

Altogether, we proved that the Discrete Fourier Transform (DFT) together with its inverse follow these two rules

$$
\begin{aligned}
& \mathcal{F}\left(\Perp \Delta_{N} f\right)=\frac{1}{N} \Delta_{\Psi_{\frac{1}{N}}}(\mathcal{F} f) \text { and } \\
& \frac{1}{N} \mathcal{F}\left(\Perp_{\frac{1}{N}} f\right)=\quad \amalg \Delta_{N}(\mathcal{F} f)
\end{aligned}
$$

as functions of $\sigma \in \mathbb{R}$, which are (5) and (6), respectively.

\section{Tricks}

We basically used two tricks in the above derivations. One is well-known. It is the so-called periodization trick, explicitly mentioned e.g. in [10-14] and in greater detail described in [15], p.61 and in [16] p.149. It is a standard technique occurring in lecture notes on signal processing (e.g. [17]), also celebrated as the link between Fourier series and Fourier transform. We also applied this trick to discrete functions when establishing the link between DTFT and DFT.

The second trick we used can be called interpolation trick. It requires generalized functions theory. We compose a loose infinite sequence of sampled values $[. . f(-2), f(-1), f(0), f(1), f(2) .$. to $(\Perp f)(t)$, i.e., to something that looks like a smooth function depending on variable $t \in \mathbb{R}$.



Figure 3: Three domains of the Fourier transform. 1: $\mathcal{F}$ maps non-discrete non-periodic functions onto each other, 2: DTFT and $\mathcal{F}_{\text {per }}$ map between discrete functions (Fourier series coefficients) and periodic functions (Fourier series), 3: The DFT, finally, maps discrete periodic functions onto discrete periodic functions. But all three transforms coincide with $\mathcal{F}$. They only differ in the kind of functions they are applied to.

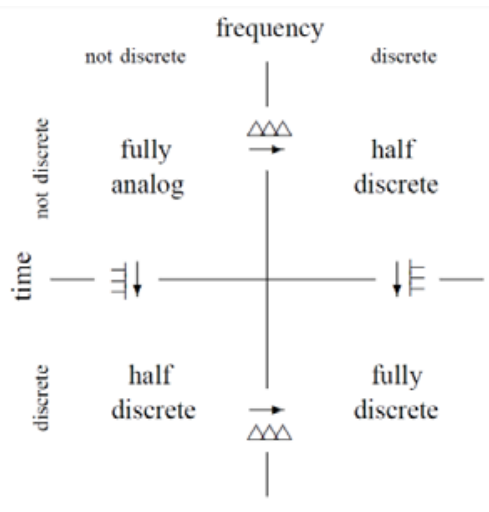

Figure 4: Three domains of the Fourier transform. 1: Fully analog domain, 2: Half discrete domain, 3: Fully discrete domain. The Fourier transform remains "captured" (is an automorphism) in each of these three domains. A duality between "fully analog" functions $f(t)$ and "fully discrete" functions $[f(0), f(1), \ldots, f(\mathrm{~N}-1)]$ allows us to use the DFT as if it were $\mathcal{F}$ provided the sampling theorem is respected twice while discretizing and periodizing $f$.

In fact, $(\Perp f)(t)$ is a smooth (infinitely differentiable) function in the generalized functions sense. The same trick is furthermore applied to finite sequences $[f(0), f(1), \ldots, f(\mathrm{~N}-1)]$, $\mathrm{N}$-tuples in other words, resulting in discrete periodic functions $\left(\Perp \aleph_{N} f\right)(t)$.

The fundamental idea behind these two tricks which can be considered dual of each other is that periodicity replaces finiteness and discreteness replaces smoothness. Note that the dual of discreteness (discrete functions) is not continuity (continuous functions) as usually taught but smoothness (smooth functions). Further details on these principles can be found in [4]. It remains to mention that both $\Perp$ and $M$ perform sampling, one in "time domain" and the other in "frequency domain" (Figure 4) which means that the classical sampling theorem must be respected twice (in time and frequency domain) otherwise aliasing effects occur; hence $f(t)$ and $\left(\Perp \aleph_{N} f\right)(t)$ would not represent the same function any more. 


\section{Diagrams}

Formulas (3)-(6) derived in this paper can moreover be depicted in diagrams such as the one in Figure 1. Additionally, both left-hand side and right-hand side in this diagram are cyclic, see Figure 2. Another most interesting aspect is that Figure 1 forms a Möbius strip if the upper left knot is identified with the lower right and lower left with the upper right. This circumstance can be interpreted in a way that tells us that it actually remains unclear whether the left-hand side is "time domain" or the right-hand side because there is no left-hand side and no right-hand side, just opposite sides.

Having a look at Figure 3 and Figure 4 one may observe furthermore that despite the fact of having only one Fourier transform $\mathcal{F}$, it is actually "captured" either on "fully analog" functions ("analog" in both time and frequency domain), on "fully discrete" functions ("discrete" in both time and frequency domain) or on "half discrete" functions, i.e., functions which are only discrete in either time or frequency domain.

\section{Naming Conventions}

The naming of $\mathcal{F}, \mathcal{F}_{p e r}$, DTFT and DFT in the literature is often not very appropriate and sometimes confusing. By looking at Table 3 and Table 4, they could be called "Fourier transform", "Fourier transform for periodic functions", "Fourier transform for discrete functions" and "Fourier transform for discrete periodic functions". However, they are all the same Fourier transform $\mathcal{F}$ but differ in the kind of functions $(f, \Perp f, \bowtie f$, $\Perp \Delta \sim f$ ) they are applied to.

An introduction of two transforms $\mathcal{F}_{\text {per }}$ and DTFT is also not advisable because they are, apart from having an inverse sign (which indicates that they are inverse to each other), the same transform (Figure 3). Most appropriately, it could be called the "Fourier Series Transform" as it switches between two Fourier series representations, i.e., between discrete functions (Fourier series coefficients) and periodic functions (Fourier series). Its two formulas should be called "Fourier Series Analysis" and "Fourier Series Synthesis" formula, as already done in many textbooks.

\section{Applications}

A main application of the above derivations lies of course in the fact that they can well be used for educational purposes, i.e., for teaching the interrelationships between Fourier series and Fourier transform and between different Fourier transform variants. Furthermore, the rules above can be fed into symbolic calculation environments such as Wolfram Mathematica [18] and/or Python SymPy [19] and by that become an indispensable toolbox for algorithm design. Recall that Poisson's summation formula allows switching from badly converging algorithms to rapidly converging ones [6].

\section{Conclusions}

It is shown that instead of four Fourier transforms, we only have one Fourier transform, the Fourier transform in the distributional sense. We found that Poisson's Summation Formula is the actual link in-between these four transform definitions and, in contrast to the Fourier-Poisson cube, we were able to explicitly write down these links in connecting formulas which do moreover rigorously hold in the tempered distributions sense.

\section{APPENDIX - Fourier Transforms}

There are mainly three ways of how to deal with the factor $2 \pi$ in Fourier transform definitions. Here, we use the so-called "unitary, ordinary frequency" Fourier transform [20] as it can be found for example in [2]. It uses 1-periodic exponential functions $e^{2 \pi i \sigma}$ rather than $2 \pi$-periodic ones $e^{i \sigma}$ and thereby yields the most symmetric results in Fourier transform pairs, e.g. $\mathcal{F} \delta=1$ and $\mathcal{F} 1=\delta$. As a result of this, "time domain" and "frequency domain" become fully equivalent.

Now, let $f(t)$ be a suitable function such that it can be Fourier transformed and let $\hat{f}(\sigma)$ be its Fourier transform. Then the following four Fourier transform variants are usually defined.

\section{Fourier Transform}

The Integral Fourier Transform (for non-discrete non-periodic functions) is defined by

$$
\begin{array}{ll}
\hat{f}(\sigma)=\int_{-\infty}^{\infty} f(t) e^{-2 \pi i \sigma t} d t & \text { Analysis } \\
f(t)=\int_{-\infty}^{\infty} \hat{f}(\sigma) e^{2 \pi i t \sigma} d \sigma & \text { Synthesis }
\end{array}
$$

for suitable $f(t)$.

\section{Fourier Transform (for periodic functions)}

The Integral Fourier Transform for (non-discrete) periodic functions, used for Fourier series analysis, is defined by

$$
\begin{array}{ll}
\hat{f}(m)=\frac{1}{T} \int_{0}^{T} f(t) e^{-2 \pi i \frac{m}{T} t} d t & \text { Analysis } \\
f(t)=\sum_{m=-\infty}^{\infty} \hat{f}(m) e^{2 \pi i \frac{t}{T} m} & \text { Synthesis }
\end{array}
$$

where (10) is the Fourier series of $f(t)$ and (9) determines its coefficients. The coefficients are discrete.

\section{Fourier Transform (for discrete functions)}

The Fourier Transform for discrete (non-periodic) functions, also called Discrete-Time Fourier Transform (DTFT), used for Fourier series synthesis, is defined by

$$
\begin{array}{ll}
\hat{f}(\sigma)=\frac{1}{T} \sum_{k=-\infty}^{\infty} f(k) e^{-2 \pi i \frac{\sigma}{T} k} & \text { Analysis } \\
f(k)=\int_{0}^{T} \hat{f}(\sigma) e^{2 \pi i \frac{k}{T} \sigma} d \sigma & \text { Synthesis }
\end{array}
$$

where (11) is a Fourier series. Hence, it is periodic but $f(t)$ itself is discrete, its samples are determined by (12).

\section{Fourier Transform (for discrete periodic functions)}

The Fourier Transform for discrete periodic functions, called Discrete Fourier Transform (DFT) is defined by

$$
\begin{array}{ll}
\hat{f}(m)=\frac{1}{N} \sum_{k=0}^{N-1} f(k) e^{-2 \pi i \frac{m}{N} k} & \text { Analysis } \\
f(k)=\sum_{m=0}^{N-1} \hat{f}(m) e^{2 \pi i \frac{k}{N} m} & \text { Synthesis }
\end{array}
$$

where both, (13) and (14), are (finite-sum) Fourier series. Thus, they are periodic and discrete, simultaneously. 


\section{References}

1. S. L. Sobolev and V. L. Vaskevich (1997), The Theory of Cubature Formulas, Mathematics and Its Applications, Vol. 415. Dordrecht, The Netherlands: Springer-Science+Business Media B.V.

2. D.W. Kammler (2007), A first course in Fourier analysis, 2nd ed. Cambridge University Press.

3. J.V. Fischer (2015), On the Duality of Discrete and Periodic Functions. Mathematics, 2015, vol. 3, no. 2, pp. 299-318, doi:10.3390/math3020299.

4. J.V. Fischer (2017), On the Duality of Regular and Local Functions. Mathematics, vol. 5, no. 3, 41, doi:10.3390/math5030041.

5. J. Fischer (1997), Anwendung der Theorie der Distributionen auf ein Problem in der Signalverarbeitung, Diploma Thesis, Ludwig-Maximillians-Universität München, Fakultät für Mathematik.

6. P.M. Woodward (1953), Probability and Information Theory, with Applications to Radar. Oxford, UK: Pergamon Press Ltd.

7. D. Brandwood (2003), Fourier Transforms in Radar and Signal Processing. Norwood, MA, USA: Artech House.

8. L. Schwartz $(1950,1951)$, Théorie des Distributions, Tome I and Tome II, Herman, Paris.

9. J. Horváth (1966), Topological Vector Spaces and Distributions. Addison-Wesley Publishing Company: Reading, MA, USA.

10. K. Gröchenig (2001), Foundations of Time-Frequency Analysis, Birkhäuser: Basel, Switzerland.

11. H.G. Feichtinger and K. Gröchenig (1997). Gabor frames and time-frequency analysis of distributions. Journal of Functional Analysis, 146.2, 464-495.

12. C. Aslangul, N. Pottier, D. Saint-James (1989), Velocity and diffusion coefficient of a random asymmetric one-dimensional hopping model, Journal de Physique, 50 (8), pp.899-921. doi 10.1051/jphys:01989005008089900.

13. S. Hartmann (2015), Quaternionic Gabor Expansion, Diploma Thesis, Technische Universität Bergakademie Freiberg, Fakultät für Mathematik und Informatik.

14. C. Cabrelli, D.G. Lee, Ursula Molter, G.E. Pfander (2017), Time-frequency shift invariance of Gabor spaces generated by integer lattices, arXiv:1705.02495

15. M.J. Lighthill (1958), An Introduction to Fourier Analysis and Generalised Functions. Cambridge, NY, USA: University Press.

16. W. Walter (1994). Einführung in die Theorie der Distributionen; BI-Wissenschaftsverlag, Bibliographisches Institut \& FA Brockhaus: Mannheim, Germany.

17. S. Quegan (1993), Signal Processing, Lecture Notes. University of Sheffield.

18. Wolfram Mathematica (2017). http://reference.wolfram.com/language/tutorial/SymbolicCalculations, accessed on October 02, 2017.

19. Python SymPy (2017).

https://en.wikipedia.org/wiki/SymPy, accessed on October 02, 2017.

20. Wikipedia Fourier Transform (2017).

https://en.wikipedia.org/wiki/Fourier\%20transform,

accessed on October 02, 2017. 